\title{
Perfektionismus und liberaler Egalitarismus
}

\author{
Ein Versuch ihrer Vermittlung
}

\author{
Von CHRISTOPH HENNING (St. Gallen)
}

In der politischen Philosophie hat sich neben dem egalitären Liberalismus nach John Rawls und seinen einerseits libertären, andererseits kommunitaristischen Kritiken eine weitere Grundposition etabliert, die „Perfektionismus“ genannt wird. Güte oder Gerechtigkeit sozialer Strukturen und sozialpolitischer Maßnahmen werden hier nicht länger an einer egalitären Verteilung von Grundgütern, einer Gewährleistung negativer Freiheiten oder einer Ermöglichung von Gemeinschaft gemessen, sondern an der Entwicklung der Fähigkeiten der Bürger. Es scheint, als gerate der liberale Egalitarismus damit endgültig ins Hintertreffen, denn dieser Ansatz wird besonders in konservativen Kreisen geschätzt. ${ }^{1}$ Zwar hat der - verwandte Fähigkeitenansatz von Amartya Sen auch das Denken über Entwicklungshilfe verändert, doch die Transformation des „aktivierenden“ Wohlfahrtsstaats in den westlichen Regionen (die jüngsten Sozialreformen haben mit einer Rhetorik der Autonomieförderung Sozialleistungen gekürzt und an Arbeitsanforderungen geknüpft) gibt dem Eindruck Recht, es seien vor allem anti-egalitäre und illiberale Versionen perfektionistischen Denkens, die sich eine praktische Wirkung zu verschaffen wissen. Vielleicht lassen liberale Autoren auch aus diesem Grund gegenüber dem perfektionistischen Denken Skepsis walten. Meine Überlegungen wollen sich dieser Skepsis nicht ergeben. Sie unternehmen vielmehr den Versuch, gegenüber der hegemonialen Lesart des Perfektionismus eine progressivere Variante zu vertreten. Dafür reicht es nicht aus, sich auf gegenwärtige philosophische Autoren zu beziehen, denn ihre Diskussion wird in gehöriger Entfernung zur Politik geführt. Zwar machen sich auch die Philosophen zum Anwalt einer Politik, die jenseits der liberalen „Neutralität“ des Staates zur Autonomieförderung beitragen soll. Doch worin genau diese bestehen soll und welche Maßnahmen im Einzelfall zu begründen wären, bleibt meist eher vage. ${ }^{2}$

1 „Perfectionist arguments are found across the contemporary American political spectrum, but are most commonly associated with neoconservative arguments." (A. W. Dzur, Liberal perfectionism and democratic participation, in: Polity, 30, 1998, 668) Konservative Vertreter sind Robert P. George (Making Men Moral. Civil Liberties and Public Morality, Oxford 1993), der Theologe John Finnis, die Ideengeschichtlerin Gertrude Himmelfarb oder Lawrence Mead (Beyond Entitlement. The Social Obligations of Citizenship, New York 1986).

2 „Governments are subject to autonomy-based duties to provide the conditions of autonomy for people who lack them.“ (J. Raz, The Morality of Freedom, Oxford 1986, 415) Zu welchem Zweck oder in 
Es gibt, anders gesagt, nicht nur eine, sondern eine ganze Palette perfektionistischer Positionen. Hier gilt es, Übersicht zu schaffen. Zunächst wird daher in einem ersten Teil dieses Aufsatzes das Spektrum des ,,philosophischen“ Perfektionismus umrissen (I). Dabei stehen als Unterscheidungskriterien das Verhältnis zum Egalitarismus (1) und zum Liberalismus (2) sowie die Bestimmung der menschlichen Natur (3) im Vordergrund. Die liberale und egalitäre Variante wird dabei auf dem Weg einer ideengeschichtlich informierten Vergegenwärtigung gewonnen. Denn die Idee, dass Menschen bestimmte Anlagen haben (individuelle Talente wie gattungsspezifische „Wesenskräfte“), deren Realisierung für ein „gutes Leben“ von Bedeutung ist, ist ja keineswegs neu. Sie wurde von Aristoteles bis zu Wilhelm von Humboldt und darüber hinaus diskutiert, und sie hatte durchaus nicht immer eine konservative Schlagseite. Es fragt sich also, ob sich nicht auch ein politischer Perfektionismus denken ließe, der das normative Erbe des liberalen Egalitarismus wahrt. Dazu kann eine Neulektüre von Karl Marx einiges beitragen (II): Marx ist ein perfektionistischer Denker, dessen Variante eine nähere Betrachtung verdient, da sie zugleich egalitär (1) und individualistisch (2) ist und auf einer durchaus originellen Anthropologie aufruht (3). Teil II löst so die in Teil I nur aufgewiesene Möglichkeit eines egalitären und liberalen Perfektionismus anhand eines Beispiels ein.

\section{Parameter der philosophischen Perfektionismusdiskussion}

Was verstehen Philosophen unter „Perfektionismus“? Zunächst ist der Begriff abzugrenzen von der alltäglichen Verwendungsweise, die damit einen Habitus des „Nie-Zufrieden-Seins“ bezeichnet. Ein perfektionistischer Maler betrachtet sein Werk ungern als vollendet und kann darum jahrelang an nur einem Gemälde arbeiten. Perfektionismus grenzt in diesem Verständnis an eine Pathologie, da das normale Leben durch ihn immer einen Schatten des Unvollkommenen erhält und die aktive Betätigung so gerade gehemmt werden kann. ${ }^{3}$ Dieses Verständnis ist nicht gemeint, ebenso wenig wie die Spekulation einer historischen Vervollkommnung des Menschengeschlechtes, wie sie etwa Condorcet vorschwebte und wie sie heute im Horizont der Gentechnik als Horrorszenario auftaucht. ${ }^{4}$ Gemeint ist vielmehr ein Verständnis des einzelnen menschlichen Lebens, das auf ein langfristiges Gelingen abzielt. Dieses wird im Englischen als Blühen (flourishing) gefasst und an die Entwicklung wünschenswerter Fähigkeiten und deren Ausübung gebunden (beispielsweise an ästhetische, intellektuelle oder körperliche Vermögen), die intrinsisch wertvoll seien. Dieses Gelingen wird als ein Gut angesehen, das insofern als „objektiv“ gilt, als momentane Empfindungen und Wünsche der Individuen nicht über die Güte der dazu nötigen Vorkehrungen entscheiden. ${ }^{5}$ Dieses objektive Gut kann, muss aber nicht vorfestgelegt sein.

welcher Höhe umverteilt werden soll, bleibt hingegen offen. Konkreter werden: G. Sher, Beyond Neutrality. Perfectionism and Politics, Cambridge 1997; und S. Wall, Liberalism, Perfectionism and Restraint, Cambridge 1998; doch erörtern sie eher abseitige Fälle wie die Gurtpflicht oder eine Steuererleichterung für Museumsbesuche.

3 Pathologisierende Wirkungen eines übersteigerten Ideals von „Selbstverwirklichung“ beschreibt: E. Illouz, Gefühle in Zeiten des Kapitalismus, Frankfurt/M. 2006, $81 \mathrm{f}$.

4 Zur Kritik vgl. Ch. Henning, Verbesserung des Menschen: Warum, und in welcher Hinsicht?, in: Philosophische Rundschau, 56 (2009), 111-129.

5 „The central perfectionist thought is that these states [knowledge and achievement, $\mathrm{CH}$ ] are good independently of any connection with happiness and that a prime moral duty is to promote them in 
Im Bereich der Ethik ist diese Abgrenzung einerseits gegen „subjektive“ Vorstellungen vom Guten gewendet, die dieses utilitaristisch als Lust (pleasure, welfare) oder Wunscherfüllung (desires, preferences) interpretieren. Dem wird ein langfristiges Gelingen („Blühen“) des menschlichen Lebens gegenübergestellt, was indes zumindest insofern im Subjekt verankert sein muss, als ein fremdbestimmtes Leben kein gutes Leben wäre. Damit ist eine Nähe zur Autonomieethik Kants gegeben, die in der Folge von Christian Wolff durchaus eine Pflicht zur eigenen Vervollkommnung kannte. ${ }^{6}$ Andererseits grenzt sich das material vorgestellte Gute aber auch von formalen Ethiken wie eben derjenigen Kants ab, in der nur der ,gute Wille“, also die Pflichtmäßigkeit einer Handlung gut ist, unter Absehung von ihren tatsächlichen Folgen. Vielmehr geht es durchaus um Folgen. Diese werden allerdings in Kategorien des „objektiv“ und nachhaltig guten Lebens und nicht in solchen der Lust, Wunscherfüllung oder Gütermaximierung ausbuchstabiert. Der Perfektionismus tritt damit das aristotelische Erbe an, war doch schon die Eudaimonia an die Ausbildung gewisser Tugenden gebunden. Gut ist es also, wenn Menschen objektive Güter verwirklichen, und diese wiederum sind „gut", weil und insofern sie zu einem nachhaltig gelingenden Leben beitragen. $\mathrm{Zu}$ solchen Gütern gehören eine ausreichende Versorgung, Gesundheit, eine erfüllende Tätigkeit, Freundschaften sowie eine Ausbildung der Fähigkeiten und Talente.

Als Erbe des Aristotelismus kann es zugleich gelten, dass die Ethik mit der Politik zusammengedacht wird: Wenn es „richtig“ ist, dass Menschen sich in diese Richtung entwickeln, dann ist es die Aufgabe der Gemeinschaft, diese Entwicklung zu ermöglichen und zu fördern. Erst dieser Gedanke der politischen Förderung ist das Ärgernis für die liberale politische Philosophie, denn der Staat scheint hier seine neutrale Sphäre verlassen oder gar einbüßen zu müssen. Die spannende Frage ist, ob und inwieweit er das tun darf. Allerdings ist mit einer Antwort darauf noch nicht gesagt, was genau der Staat eigentlich tun soll. Es stellen sich an dieser Stelle also viele Fragen, die sich innerhalb des perfektionistischen Paradigmas auf verschiedene Weise beantworten lassen. Sie betreffen zunächst die Frage nach Verteilungskriterien einerseits (1), dem Entscheidungsspielraum der Individuen andererseits (2). Mitverhandelt wird dabei stets das Verständnis der menschlichen Natur, die ja das stellt, was entwickelt werden soll (3). Anhand dieser Dimensionen lassen sich ganz verschiedene Perfektionismen unterscheiden.

1. Das Verhältnis zum Egalitarismus. Grundsätzlich lässt sich der Perfektionismus sowohl egalitär als auch antiegalitär deuten, wobei letztere Deutung momentan dominant sein dürfte. ${ }^{7}$ Diese Unterschiede hinsichtlich der verteilungstheoretischen Implikationen beruhen nicht allein auf externen Werthaltungen, sondern auch darauf, wie der Perfektionismus intern konzipiert wird. Geht es um die Förderung von menschlicher Exzellenz, so kann entweder allen das gleiche Recht auf die Entwicklung ihrer Anlagen zugesprochen werden, oder es werden nach dem Maxi-Max-Prinzip diejenigen am meisten gefördert, die das höchste Potenzial aufweisen. John Rawls deutete den Perfektionismus in der zweiten, nietzscheanischen Lesart - und

oneself and in others.“ (Th. Hurka, Perfectionism, in: Routledge Encyclopedia of Philosophy, London 1998)

6 „Baue deine Gemüts- und Leibeskräfte zur Tauglichkeit für alle Zwecke an.“ (I. Kant, Metaphysik der Sitten, A 392)

7 Thomas Hurka (Perfectionism, Oxford 1993, 80) bezeichnet Thomas Nagel und Derek Parfit als nicht-egalitäre Vertreter. Ein älteres Gegenbeispiel ist: V. Haksar, Equality, Liberty, and Perfectionism, Oxford 1979. 
lehnte ihn daher ab. ${ }^{8}$ Die Annahme ist hier folgende: Demjenigen, der die besten Anlagen hat, stehen die meisten Ressourcen zu. Das bedeutet praktisch: Wenn Arme wenig können, würden sie dadurch noch ärmer. Das kommt der dominanten marktwirtschaftlichen Leistungsideologie recht nahe: Eine Verkümmerung der Anlagen wird hier oft nicht als Wirkung, sondern eher als Ursache der Einkommensschwäche gedeutet (versteckt wird dieser Naturalismus in dem Scheinbegriff der „earning capacity“). Daraus kann ein Teufelskreis entstehen, wenn scheinbar weniger „entwickelte“ Menschen (,bildungsferne Schichten“) nun gar nicht mehr die Möglichkeit zur Entwicklung bekommen. In dieser Konzeption wird Perfektion wie bei Nietzsche als ein Projekt der Eliten vorgestellt, die es vermögen, in Absonderung von der Gemeinschaft ihre menschlichen Anlagen zu vervollkommnen. ${ }^{9}$

Doch diese Lesart ist keineswegs notwendig, denn die wenigsten würden wohl privaten Reichtum mit „menschlicher Entwicklung“ gleichsetzen. Die Lesart ergibt sich erst aus einem Verständnis charakterlicher Entwicklung, welches diese in atomistischer Abtrennung von den anderen, ja in Entgegensetzung zu den anderen - dem Pöbel, den „Vielen“ - denkt (zur Fundierung im Menschenbild siehe I.3). Rousseaus Émile beispielsweise muss von dem Einfluss anderer Menschen systematisch abgeschirmt werden, um sich selbst in Fülle entwickeln zu können. Die Entwicklung kann dann nur eine sein, die als ,aus sich rollendes Rad“ von der Einbindung in Gemeinschaften abgelöst ist. ${ }^{10}$ Nicht zufällig wird im Émile als Lektüre Robinson Crusoe empfohlen. Nur eine solch vorsoziale Deutung (die bei Rousseau später wieder aufgehoben wird) kann wollen, dass die Hochkultur der Wenigen auf Kosten der Vielen erkauft wird. Die Frage für den Perfektionismus ist nun nicht, ob eine solche Verteilung „gerecht“" wäre, solange Gerechtigkeit noch unabhängig von der menschlichen Entwicklung begriffen wird.

Die Frage für Perfektionisten ist vielmehr, ob eine solche Kultur überhaupt objektiv, das heißt im Hinblick auf eine blühende menschliche Entwicklung, gut wäre. Hätte sich das derart entwickelte Individuum wirklich ,perfektioniert“, oder wäre es nicht vielmehr verarmt und, wie das Leben Nietzsches selbst vielleicht am besten zeigt, der Möglichkeiten eines nachhaltigen Gelingens beraubt? Schauen wir also, ob man den Perfektionismus nicht auch egalitär fassen kann. Ich meine, es gibt eine ganze Reihe starker Argumente für eine egalitäre Interpretation, die man im Laufe der perfektionistischen „Tradition“ immer wieder findet. ${ }^{11}$ Diese Punkte werden hier hervorgehoben, weil die andere (Maxi-Max-)Deutung in der philosophischen und politischen Diskussion ohnehin stark vertreten ist. Welche Argumente sind dies?

Das erste Argument läuft über eine Umkehr der Beweislast. Wer Verwirklichungschancen einengen will, muss angeben, mit welchem Recht andere Menschen von diesen Chancen ausgeschlossen werden. Klassische Autoren wie Rousseau, Kant und Adam Smith gingen davon aus, dass die natürliche Ausstattung der Menschen nicht derart voneinander abweiche, dass sich damit eine systematische Ungleichbehandlung ganzer Gruppen rechtfertigen las-

8 J. Rawls, Theorie der Gerechtigkeit, Frankfurt/M. 1975, $360 \mathrm{ff}$.

9 Siehe etwa Ch. Murray, Human Accomplishment. The Pursuit of Excellence in the Arts and Sciences 800 B. C. to 1950, New York 2003; vgl. Th. Hurka, Nietzsche: Perfectionist, in: B. Leiter u. N. Sinhababu (Hg.), Nietzsche and Morality, Oxford 2007, 9-31.

10 „Unschuld ist das Kind und Vergessen, ein Neubeginnen, ein Spiel, ein aus sich rollendes Rad.“ (F. Nietzsche, Also sprach Zarathustra, in: ders., Sämtliche Werke, KSA, Bd. 4, München/New York 1980, 31)

11 Die ,central tradition“ in Europa war perfektionistisch (R. P. George, Making Men Moral, a. a. O., 19 ff.), nur eben nicht immer anti-egalitär. 
se. (Ein weiteres klassisches Argument, heute vertreten von Hillel Steiner und Philipp van Parijs, besagt, dass die natürlichen und sozialen Ressourcen allen gehören und deswegen niemand von ihrer Nutzung ausgeschlossen werden darf. Dies betrifft jedoch nicht nur den Perfektionismus.) Da vielmehr erst die soziale Ungleichbehandlung dieser Gruppen bewirke, dass einzelne ihrer Mitglieder zu einem gegebenen Moment ihre Talente unterschiedlich weit entwickelt haben, ist dies gerade kein Argument für eine weitere Ungleichbehandlung, sondern ein starkes für deren Aufhebung. Wenn heute soziale Unterschiede eher mit natürlichen Unterschieden gerechtfertigt werden, steht dahinter weniger eine neue „wissenschaftliche“ Erkenntnis, sondern eher ein politischer Gewöhnungsprozess. ${ }^{12}$ Dieser ist überaus kontingent, und es gilt, ihn wieder stärker zu hinterfragen.

Das zweite Argument ist republikanisch: Wenn Menschen für ein gelingendes Leben auf eine Gemeinschaft angewiesen sind, die sie fördert, wertschätzt und versteht, muss auch diese Gemeinschaft selbst gelingen. Das kann sie nur, wenn die Unterschiede zwischen den Einzelnen nicht zu groß sind. Kann ein Armer die Reichen kaufen, so sind weder der Arme noch der Reiche dazu in der Lage, „gut“ zusammenzuleben. (Und es wäre seltsam, hier die „Kultur" vorzuschieben, als habe sie nichts damit zu tun: Kultur ist das „Wie“ des Miteinander, es kann daher keine „Kultur des Miteinander“ geben, wo es kein Miteinander gibt.) Das war für Aristoteles oder Rousseau selbstverständlich, und neuere Philosophien haben daran erinnert. ${ }^{13}$ Soziale Ungleichheit darf ein gewisses Maß nicht überschreiten, bei Strafe moralischer oder gar gesundheitlicher Schäden der Bürger. ${ }^{14}$ Statt Armut als mangelnde Entwicklung zu rechtfertigen, gilt es vielmehr, materielle Bedingungen für gleiche Entwicklungschancen aller Bürger zu schaffen.

Zwar ließe sich diese Solidarität noch gruppenegoistisch einengen, aber nur mit Not. Denn drittens tendiert eine Gesellschaft, die auf Misstrauen zwischen ihren Gruppierungen beruht, zu Faktion und sozialem Unfrieden. Dieser wirkt sich negativ auf die Lebensqualität aller Einzelnen, auch der Reichsten, und damit auf ihre Entwicklungsmöglichkeiten aus. Sozialer Friede bedarf keineswegs der Liebe zwischen allen Bürgern, wohl aber ihrer Gleichbehandlung. ${ }^{15}$ Dieses republikanische Argument der Nichtdiskriminierung von (Rand-)Gruppen hat für die Frage der Aneignungsformen gesellschaftlich erwirtschafteten Reichtums ebenfalls eine hohe Relevanz.

Viertens lässt sich utilitaristisch argumentieren, dass mit jeder zusätzlichen Einheit eines verteilbaren Gutes der geschaffene Grenznutzen auch ,in terms of perfection“ abnehme, wenn ein Empfänger bereits viel erhalten hat. Der Perfektionsnutzen ist bei Schlechtergestellten wesentlich höher. Dieses Argument wurde bereits in der Bewegung, die ich „Linksbenthamianismus“ nennen möchte, prominent gemacht. ${ }^{16}$ Ein Beispiel: Ein Maler malt nicht besser,

12 Zur Preisgabe des Egalitarismus in der Politik vgl. M. J. Thompson, The Politics of Inequality. A Political History of the Idea of Inequality in America, New York 2007; in der politischen Ökonomie: S. J. Peart u. D. M. Levy, The „Vanity of the Philosopher“. From Equality to Hierarchy in PostClassical Economics, Ann Arbor 2005. Der anglophone „brute-luck“-Egalitarismus vollzog eine ähnliche Naturalisierung: Soziale Unterschiede werden nicht mehr auf soziale Strukturen, sondern auf eine „Naturlotterie“ der Talente zurückgeführt.

13 R. H. Tawney, Equality, London 1931; A. Gutman, Liberal Equality, Cambridge 1980; E. Anderson, How Should Egalitarians Cope with Market Risks?, in: Theoretical Inquiries in Law, 9.1 (2008), 61-92.

14 R. G. Wilkinson, The Impact of Inequality: How to Make Sick Societies Healthier, London 2005.

15 „You don't have to live next to me, just give me my equality.“( N. Simone, Mississipi Goddamn, 1963)

16 So bei: W. Thompson, An Inquiry into the Principles of the Distribution of Wealth Most Conducive to Human Happiness, 2 Bde., London 1824. 
wenn er seine Bilder noch teurer verkauft. Zwar mag ein ausreichendes Stipendium seine Kreativität stark anspornen, doch spätestens die zweite verdiente Million dürfte sich kaum noch spürbar auf die Güte der gemalten Bilder auswirken. Wohl aber können viele Andere malen lernen (und vielleicht sogar gut), wenn sie dafür eine Freistellung und entsprechende Ermunterung bekommen. Können sie dies tun, ist das natürlich für ihre eigene Entwicklung gut.

Aber nicht nur für diese: Ein fünftes Argument geht davon aus, dass der Ausschluss einiger Menschen von der Ermöglichungspolitik deren Talente verschwenden würde. Damit wird nicht nur ihr eigenes Entwicklungsglück, sondern auch das der anderen eingeschränkt, ist doch nach W. v. Humboldt neben der Freiheit vor allem die ,Mannigfaltigkeit der Situationen“ entscheidend für die menschliche Entwicklung. ${ }^{17}$ Nehmen wir das Beispiel der Kunst: Je weniger Künstler tätig sind und je unfreier sie in ihrer Gestaltung sind, desto ärmer die Kunst. Je zahlreicher und unabhängiger dagegen die Künstler, desto bunter die Kunst. In einer arbeitsteiligen Gesellschaft profitieren alle von allen, wenn auch nicht immer monetär. Nicht nur die Gesellschaft insgesamt, auch ihre Mitglieder erführen also einen Verlust in ihrer Entwicklung, wenn Maler, Musiker oder sonstige Talente nicht zum Zuge kämen. Dieses Argument funktioniert also auch dann, wenn Perfektion ,akteurzentriert“ begriffen wird. Soll Perfektion hingegen insgesamt (,,akteurneutral“) maximiert werden, ist eine Ungleichverteilung ohnehin wenig sinnvoll. ${ }^{18}$

Ein abschließender sechster Punkt ist in der Ästhetik zu verorten. Wenn kulturelle Güter autistisch, das heißt in Absonderung von den Anderen, produziert und konsumiert werden (als positionale, mithin sozial ausschließende Güter), dann geht ihr intrinsischer Wert zurück, der ja für die Entwicklung elementar ist. Man denke an die Musik: Wenn diese - wofür an dieser Stelle nicht eigens argumentiert werden soll - intrinsisch vergemeinschaftend ist, wie es Schillers Ode an die Freude exemplarisch ausdrückt, dann ist eine privatistische und sektenhaft-elitäre Musikpraxis für den Perfektionismus weniger wertvoll als eine (wie auch immer gestaltete) gemeinschaftliche. Obzwar dieser Gedanke kühn erscheint, findet er sich bei namhaften Autoren wieder. ${ }^{19}$

2. Das Maß der individuellen Freiheit. Die zweite Dimension, in der sich Varianten des Perfektionismus unterscheiden, ist ihr Grad an Liberalität. Woher kommt es, dass der Perfektionismus häufig als Gegner des Liberalismus wahrgenommen wird? Wie der dritte Abschnitt zeigen wird, kann die Illiberalität aus einer bestimmten Vorstellung von Entwicklung resultieren, die allerdings nicht die einzig denkbare ist. Aber zunächst zum Augenschein: Versteht man unter individuellen Freiheitsrechten die Gewährleistung einer Privatsphäre, eines Schutzraumes vor Eingriffen Anderer oder der Gemeinschaft, einerlei, was dort geschieht ${ }^{20}$, so sieht die Vorstellung, es gäbe objektive Ziele, an denen Personen zu messen seien, zunächst illi-

17 W. von Humboldt, Gesammelte Werke, Bd. VII, Berlin 1852, 10.

18 Nach Hurka (Perfectionism, a. a. O., 55 ff.) geht es dem Perfektionismus um die größte Entwicklung der größten Zahl.

19 Adorno hatte zwar Sympathien für Schönbergs Elitismus, doch hätte er positionale Musikpraxen wohl „unwahr“ genannt. Ähnlich T. H. Green (Prolegomena to Ethics, Oxford 1883, § 381): „Under such conditions much occupation with music might imply indifference to claims of the human soul which must be satisfied in order to the achievement of a life in which the value of music could be actualised.“

20 Für Hayek ist Freiheit der „Zustand, in dem ein Mensch nicht dem willkürlichen Zwang durch den Willen eines anderen oder anderer unterworfen ist“ (Die Verfassung der Freiheit, Tübingen 1991, 14). Sie kann, muss aber nicht politisch genutzt werden. 
beral aus. Denn nutzen Individuen ihre Freiheitsräume nicht oder falsch, können sie das Ziel einer Entwicklung verfehlen. Wird eine Entwicklung zur bindenden Auflage gemacht, kann dies für die Gemeinschaft eine Legitimation sein, sie auch gegen den Willen der Individuen zu erzwingen. So hatten die Policeywissenschaften des 18. Jahrhunderts, die wir heute primär aus der Kritik her kennen, die Kant und Humboldt an ihnen geübt haben, deswegen solch paternalistische Tendenzen, weil sie eine Entwicklung von oben her betrieben. Aber wirklich ungewöhnlich ist dieser Gedanke nicht: An die Schulpflicht etwa haben wir uns ja gewöhnt. Darf man also im Interesse der Ermöglichung von Entwicklung Freiheit einschränken?

Diese schwierige Frage wird manchmal mit der Unterscheidung zwischen (negativer) Freiheit und (positiver) Autonomie zu beantworten versucht. ${ }^{21}$ Doch ist dies eine ausreichende Antwort? Systematisch macht es zunächst einen Unterschied, welche Rolle die Autonomie für die angestrebte Entwicklung spielt: Ist das Führen eines selbstbestimmten Lebens ein Gut unter vielen, könnte ihr Fehlen möglicherweise durch andere Güter aufgewogen werden. Selbst wenn Autonomie als das „höchste“ Gut im Sinne einer Werthierarchie gilt, ähnlich wie in der Bedürfnispyramide Maslows, ließen sich „relativ“ perfekte Zustände denken, die vorautonom zu Stande gekommen wären. ${ }^{22}$ Ein Beispiel: Wenn meine Eltern mich in einen Beruf pressen, der mir ein gutes Einkommen, viele Kontakte und soziales Prestige eröffnet, könnte dies als „objektiv gut“ begriffen werden, selbst wenn ich nicht ein Gran Selbstbestimmung an den Tag lege, sondern nur das tue, was von mir erwartet wird - vorausgesetzt, Autonomie ist ein Gut unter vielen, das aufgewogen werden kann. Meine negative Freiheit wird jedenfalls nicht verletzt. Gilt Autonomie jedoch als konstitutiv, so wird eine Perfektion von außen schwierig: Ich selbst muss es sein, der diese Prozesse will und in Gang setzt. Ein Eingriff kann die Entwicklung eines Individuums verschlechtern, wenn ihm fremde Ziele imputiert werden. Wenn ein Staat für mich bestimmte Güter schafft, womöglich gar in Austausch gegen die Aufgabe weitergehender, etwa partizipativer Freiheiten, wäre das aus dieser Sicht keine echte „Perfektion“. Sie wäre als solche erst zu begreifen, wenn sich die Subjekte zugleich als Urheber dieser Vergünstigungen verstehen können (etwa durch politische Mitspracherechte). Im Bild Maslows: Das Fehlen der „Spitze“ wirkte sich noch auf die Qualität der untersten Güter aus. Das Brot in Freiheit schmeckt aus dieser Sicht besser als der Kuchen in Sklaverei; wohlgenährte Sklaven bleiben eben Sklaven.

Es scheint bisher so auszusehen, dass mehr Autonomie immer „besser“" ist. Doch hier versteckt sich ein Problem. Die Verhältnisbestimmung zwischen Autonomie und negativer Freiheit wird in dem Moment erschwert, wo - wie im politischen Perfektionismus - eine Autonomieförderung angestrebt wird. Die Schwelle für ein autonomes Leben kann unterschiedlich hoch gelegt werden. Daraus entsteht nun das Paradox, dass Eingriffe in die Freiheit desto eher gerechtfertigt werden können, je höher die Schwelle der Autonomie liegt. Gehört zu einem Verständnis von Autonomie nicht nur, einen Beruf zu ergreifen und Freundschaften zu knüpfen, sondern auch - überspitzt gesagt -, bestimmte der Oberschicht wohlgefällige Frisuren zu tragen (da erst diese den Zugang zu höheren Kreisen ermöglichen) oder Mozart zu hören (da Mozarts Musik „exzellenter“ sei als Muzak oder jeder andere Musikgeschmack

21 Raz etwa setzt die Fähigkeit zu einem autonomen Leben mit der „positiven Freiheit“ gleich (The Morality of Freedom, a. a. O., 408). „(Significantly) autonomous persons are those who can shape their life and determine its course." (Ebd., 154)

22 Maslow unterscheidet aufsteigend körperliche Grundbedürfnisse, Sicherheitsbedürfnisse, Sozialitätsbedürfnisse, Anerkennungsbedürfnisse und an der Spitze ein solches nach Selbstverwirklichung (A. H. Maslow, A Theory of Human Motivation, in: Psychological Review, 50, 1943, 370-396). Für Raz (The Morality of Freedom, a. a. O.) ist Autonomie in modernen Gesellschaften unverzichtbar. $\mathrm{Ob}$ sie jedoch einen kontextunabhängigen Wert hat, bleibt bei ihm ungreifbar. 
als nicht selbstgewählt gilt) ${ }^{23}$, so ist die „Förderung“ dieser höherstufigen Autonomie wohl nur um den Preis eines Eingriffes in die Privatsphäre zu haben.

Ein ,autonomiefunktionaler“ Eingriff in die Freiheitsrechte bringt also Schwierigkeiten mit sich. Er gleicht einer Wette, die drei Unsicherheiten enthält: Die Akteure müssen behaupten, dass der momentane Eingriff in die negativen Freiheitsrechte langfristig (1) zu einer höheren Autonomie verhilft, als es die ist, die durch den Eingriff verletzt wird (2), und er vom Subjekt retrospektiv als wünschenswert anerkannt werden wird (3). Wird eine dieser Bedingungen nicht erfüllt, wäre die erreichte Veränderung de facto eine Verschlechterung. „Autonomiefunktionale“ Eingriffe bleiben folglich hypothetisch und riskant. John Stuart Mill hat sie darum für Erwachsene prinzipiell abgelehnt, denn die negative Freiheit würde so nicht mehr aus Prinzip geschützt, sondern sie dürfte im Interesse eines erwarteten höheren Gesamtnutzens (in Begriffen einer „character formation“) verletzt werden. Dies verstieße gegen das liberale Grundprinzip, dass Eingriffe nur durch ein Schadensverbot gerechtfertigt werden können. ${ }^{24}$ Über den Grad der Liberalität eines Perfektionismuskonzeptes entscheidet somit nicht schon der Wert, der der Autonomie gegeben wird (die ja verschieden bestimmt werden kann), sondern vor allem der Rang der negativen Freiheit - auch dann, wenn diese nicht zur Entwicklung genutzt wird. Mit anderen Worten: Autonomiefördernde Politik ist nicht automatisch schon liberal.

3. Vorstellungen von der menschliche Natur. Perfektionistische Konzepte sind Entwicklungsmodelle. Das wirft schließlich die dritte Frage auf, was eigentlich entwickelt werden soll. Dieses potenzielle Etwas kann nicht nichts sein, sonst wäre es keine Entwicklung - die Aktualisierung setzt eine Potenz, eine Anlage voraus. ${ }^{25}$ Die Frage ist jedoch, wie diese zu denken ist: als vorfestgelegte endliche Zielvorgabe, wie es beispielsweise in den Lebensformen des Aristoteles (vita activa, vita contemplativa) angedacht wird, oder als offener und damit prinzipiell unendlicher Prozess, wie etwa bei Emerson?

Beide Denkmodelle haben ihre Vorteile. Im ersten Fall wäre recht klar festzustellen, bis zu welchem Grad eine Person ein Telos, eine feldspezifische „Exzellenz“, bereits erreicht hätte. Es gäbe Vorbilder und Rollenmuster sowie klare Kriterien, wann diese erreicht sind. Für politische Applikationen hinsichtlich von Erziehungsidealen oder für Verteilungsfragen wäre das ein Vorteil. Doch hat ein teleologisches Modell auch Nachteile. Der erste praktische Nachteil wäre, dass für individuelle Unterschiede wenig Raum bliebe, da die Ziele für alle Menschen dieselben wären. Ich hätte nur die Wahl zwischen verschiedenen Zielen, doch sie alle wären vorfestgelegt, und das Individuum könnte sich darum beengt fühlen. ${ }^{26}$ Damit ließe sich zweitens ein dirigistisches Einwirken von Außen rechtfertigen. Denn unter Maß-

23 D. Parfit, Overpopulation and the Quality of Life [1986], zuletzt in: E. Ryberg u. T. Tännsjö (Hg.), The Repugnant Conclusion. Essays on Population Ethics, Dordrecht 2004, 7-22.

24 Vgl. Ch. Henning, John Stuart Mill: Ein Perfektionist? Moralischer Fortschritt und Philosophie der Arbeit bei einem Klassiker des Liberalismus, Universität St. Gallen, Scala Discussion Paper, 18 (2009).

25 ,[D]ein innerstes Bedürfnis erzeugt und nährt den Wunsch, die Anlagen, die in dir zum Guten und Schönen ruhen mögen, sie seien körperlich oder geistig, immer mehr zu entwickeln und auszubilden." (J. W. Goethe, Wilhelm Meisters Lehrjahre [1795], in: ders., Werke, Hamburger Ausgabe, Bd. VII, München 1998, 276)

26 Anders sieht dies: M. Hampe, Vervollkommnung des Individuums und Endgültigkeit der Metaphysik. Richard Rortys Metaphysikkritik und die Philosophie des Pragmatismus, in: U. J. Wenzel (Hg.), Vom Ersten und Letzten. Positionen der Metaphysik in der Gegenwartsphilosophie, Frankfurt/M. 1998, 153-176. 
gabe eines bestimmten Zieles kann ich auf dem „rechten Weg“ sein oder davon abkommen, was dann Sanktionen von außen erlauben würde. Ein theoretischer Nachteil wäre drittens, dass erkenntnistheoretisch schwer auszumachen ist, wie man ein Wissen über die präexistente menschliche „Natur“ soll erlangen können, wenn nicht an eben den Zielen, auf die die Menschen ausgerichtet werden - also an der Aktualität. Man erkennt auf diese Weise keine Möglichkeiten, sondern man normativiert Wirklichkeiten. Eine solche Ausrichtung an vorfestgelegten und bereits beispielhaft „ausgewickelten“ Zielen könnte viertens denkbare weitere Entwicklungsmöglichkeiten der menschlichen Natur beschneiden, sollte diese sich als reichhaltiger erweisen, als der historisch jeweilige „Möglichkeitssinn“ (Robert Musil) es zulässt. Man könnte, anders gesagt, nicht mehr „experimentell leben“ (John Stuart Mill). Wenn natural law-Theoretiker vor amerikanischen Gerichten mit diesem Argument gleichgeschlechtliche Ehen verhindern wollten, ist diese Möglichkeit wirklich geworden. ${ }^{27}$

Die alternative Deutung liest die Potenzialität darum mit Nietzsche als eine „nichtfestgestellte“. Bereits Rousseaus Begriff der ,perfectibilité“ meinte ja, dass sich ein Mensch in alle denkbaren Richtungen entwickeln könne. Dabei würden weniger präexistente Gattungseigenschaften als eben individuelle Eigenheiten verwirklicht. Ein dirigistischer Einfluss von außen und ein Festlegen auf schon bestehende Muster kann in dieser Sicht ferngehalten werden. Das ist Rousseaus Idee „negativer Erziehung“, die unter anderem von Schleiermacher und J. S. Mill aufgegriffen wurde. ${ }^{28}$ Sie erlaubt das Wegräumen von Hindernissen, doch sie untersagt Versuche einer direkt positiven Einflussnahme. Ob diese Individualität als bereits zuvor keimhaft bestehend gedacht wird wie bei Leibniz, oder ob sie sich erst im Prozess des ständigen Suchens und sich Veränderns herausbilden soll, macht vor allem für die Praxis einen Unterschied. Der Anti-Essenzialismus (das Modell ohne „Potenzialität“) hätte den Vorteil, dass bei der Flexibilität einer Person kaum mehr Grenzen erkennbar sind - sie kann ihre Lebenspläne gegebenenfalls immer ändern. In diesem Fall gibt es keine individuelle „Natur“, der man treu bleiben könnte, man könnte sich unbegrenzt umkrempeln. Es gäbe kein Maß der „Vollkommenheit" mehr, und Selbstverwirklichung bliebe ein unabschließbarer Prozess. ${ }^{29}$ Dies ist allerdings ambivalent: Es kann Individuen unabhängiger machen, nimmt ihnen aber auch den Halt, irgendwo innehalten und verweilen sowie mit dem Erreichten eine Anerkennung anderer erwarten zu können. Ich meine, auch aus Gründen der Konsistenz, dass die Annahme einer vorangehenden ,inneren Natur“ sehr viel mehr Sinn macht. ${ }^{30}$ Nimmt man eine innere Natur an und ändert sich, hätte man sich über seine „wahre“ Natur getäuscht, es entstünde ein biographischer Bruch, und das entspricht dem häufigen phänomenalen Befund. Mit der Voraussetzung einer ,inneren Natur" lässt sich zudem eine Kritik an sozialen Zumutungen besser artikulieren, denn ohne sie ist eine Grenze des Machbaren nur schlecht zu markie-

27 Wie im Fall Evans versus Romer in den USA. Dazu N. Bamforth u. D. A. Richards, Patriarchal Religion, Religion, Sexuality, and Gender. A Critique of New Natural Law, Cambridge 2008.

28 R. Bolle, Jean Jacques Rousseau: Das Prinzip der Vervollkommnung des Menschen durch Erziehung und die Frage nach dem Zusammenhang von Freiheit, Glück und Identität, Münster 1995, $107 \mathrm{ff}$.

29 So etwa R. Jaeggi, Entfremdung. Zur Aktualität eines sozialphilosophischen Problems, Frankfurt/M. 2005.

30 Ch. Menke (Innere Natur und soziale Normativität. Die Idee der Selbstverwirklichung, in: Die kulturellen Werte Europas, hg. v. H. Joas u. K. Wiegandt, Frankfurt/M. 2005, 304-352) verteidigt ebenfalls die „innere Natur“ (317), indem er sie in die Potenz setzt (,seine Möglichkeit, sein Vermögen“, 329, Fn.). Für diese Position scheinen mir auch die gewichtigen Einwände Manfred Franks gegen das Modell einer „,intersubjektiven“ Konstitution von Subjektivität zu sprechen, das stets schon voraussetzt, was zu erklären ist. 
ren. ${ }^{31}$ Trotz dieser praktischen Differenzen wird dieser Unterschied auf Grund des erkenntnistheoretischen Problems hinsichtlich der menschlichen Natur innerhalb individualistisch-offen ausgerichteter Perfektionismen nicht immer gemacht.

Schließlich sei darauf hingewiesen, wie die beiden ersten Dimensionen mit der dritten Dimension zusammenhängen. Traditionell egalitäre Positionen haben meist vor dem Hintergrund einer gemeinsamen menschlichen Natur argumentiert (im Kontrast dazu gehen luck-Egalitaristen von einer natürlichen Ungleichheit aus), während liberale Positionen in der Regel mit einem unabgeschlossenen Bild der Entwicklung argumentierten (gefasst im Bild des „Fortschritts“). Das muss nicht zusammengehen, allerdings ist es keineswegs ausgeschlossen, beides zusammen zu denken. Es macht durchaus Sinn zu behaupten, dass die gemeinsame menschliche Natur der Menschen gerade in der Anlage zu ganz verschiedenen Lebensweisen besteht - Menschen können eben Dinge tun, die vor ihnen noch niemand getan hat. Die „menschliche Natur“ hindert sie nicht daran, es besteht folglich kein Grund, nur aus liberalem Interesse Anti-Essentialist zu werden.

Fassen wir nun die Vergleichsparameter des ersten Teils zusammen, so kann ein Perfektionismus egalitaristisch oder anti-egalitaristisch auftreten, er kann liberal sein oder illiberal, und er kann geschlossen (teleologisch) sein, das heißt bestimmte Ziele vorher festlegen, oder Entwicklung zieloffen bestimmen (und damit den Individualismus prämieren) - alles dies nicht aus externen Gründen, sondern auf Grund der jeweiligen internen Bestimmung des Perfektionismus. Angesichts dieser Varianz ist es wenig verwunderlich, dass der Perfektionismus in der Philosophie der Gegenwart hinsichtlich politischer Forderungen selten konkret wird. Der folgende zweite Teil will dieser Offenheit entgegentreten, indem eine konsistente Version des Perfektionismus aufgezeigt wird, die in eine progressivere, eben individualistische und egalitäre Richtung geht. Dies geschieht am Beispiel des Perfektionismus von Karl Marx.

\section{Perfektionismus bei Karl Marx}

An die Stelle der alten bürgerlichen Gesellschaft mit ihren Klassen und Klassengegensätzen tritt eine Assoziation, worin die freie Entwicklung eines jeden die Bedingung für die freie Entwicklung aller ist. ${ }^{32}$

Karl Marx

Auch wenn Karl Marx keine systematische Abhandlung über „den Menschen“ oder „,die Ethik“ geschrieben hat, ist seine Version des Perfektionismus nicht schwer zu erfassen. Durch seine Werke ziehen sich Stellen, die im Sinne der Sozialphilosophie der Aufklärung davon ausgehen, dass das Ziel der Vergesellschaftung die „,volle und freie Entwicklung jedes Individuums“ sei (MEW 23, 618). An diesem Ziel sind Gesellschaften für ihn folglich zu messen. Die Aufklärung hatte an dieser Stelle mit dem Glück argumentiert und damit der französischen und amerikanischen Revolution Argumente geliefert. Wenn ein Staat das Glück der Bürger, um dessentwillen

31 Vgl. J. Marks, Perfection and Disharmony in the Thought of Jean-Jacques Rousseau, Cambridge 2005.

32 MEW 4, 482. Zitiert nach der MEW-Ausgabe (Marx-Engels-Werke, Berlin 1956 ff.), mit Angabe von Band und Seitenzahl. 
er da sei, nicht gewährleiste, sei dies Anlass für grundlegende Änderungen. ${ }^{33}$ Nun ist es umstritten, was mit „Glück“ genauer gemeint sei: eine Lockesche Eigentumsapologie, ein Humescher Sensualismus, eine republikanische Tugendformel oder die aristotelische Eudaimonia. ${ }^{34}$ Bei Marx kommt mit der „Entwicklung“ vor allem der letzte Kandidat in Betracht, allerdings in einer mit radikal-aufklärerischen Elementen durchsetzten Form. Marx ist auch im ethischen Bereich zunächst Aristoteliker. ${ }^{35}$ Er geht wie Aristoteles davon aus, dass Menschen Anlagen zu Fähigkeiten haben, die sich durch entsprechende Betätigung verwirklichen lassen (oder sonst verkümmern), und dass es für Menschen „gut“ ist, wenn sie dies tun. Auch die Bestimmung des Menschen als „Zôon politikon“ teilt Marx mit Aristoteles (MEW 113, 616). Doch es gibt gegenüber Aristoteles zugleich drei wichtige Unterschiede in der Ausformulierung dieses Konzeptes. Denn es finden sich bei Marx, orientiert an obiger Systematik, ein Egalitarismus (1) und Individualismus (2) sowie eine zieloffene menschliche Natur (3).

1. Marx' egalitärer Perfektionismus. Marx folgt dem humanistischen Bildungsbegriff, der Reichtum nicht verkürzt in Geld, sondern als „Entwicklung des Reichtums der menschlichen Natur" vorstellt (MEW 26.2, 111). Das ähnelt nicht nur von weitem dem Fähigkeiten-Ansatz von Amartya Sen. ${ }^{36}$ Die Menschen sollen sich möglichst ,allseitig“ ausbilden (MEW 3, 424 u. ö.), doch die kapitalistische Produktionsweise schneide die Menschen von ihren Entfaltungsmöglichkeiten ab (,entfremde“ sie), indem die Lohnarbeit sie vereinseitige, verdumme und verarme (MEW 40, 514 ff.), und indem das Geld den menschlichen Reichtum verdingliche und seine Verwirklichung nutzenegoistisch einschränke. Berühmt ist die - nicht ganz ernst gemeinte - Vision, dass es den Menschen in der ansonsten unbestimmt bleibenden „kommunistischen Gesellschaft“ freistünde, „heute dies, morgen jenes zu tun, morgens zu jagen, nachmittags zu fischen, abends Viehzucht zu treiben, nach dem Essen zu kritisieren, wie ich gerade Lust habe“" (MEW 3, 33). Die menschliche Natur soll sich also nicht nur gesellschaftlich auswickeln, indem Aufgaben arbeitsteilig aufgeteilt werden - das wäre ein Exzellenzkollektivismus, wie er in Platons Politeia angedacht wurde. ${ }^{37}$ Vielmehr soll auch jeder Einzelne verschiedene Facetten in sich verwirklichen. Damit werde, so der junge Marx emphatisch, die Trennung zwischen menschlichem Wesen und Individuum überwunden (,menschliche Emanzipation"; MEW 1, 370). Das ist weder eine Verschmelzungsutopie mit metaphysischen Über-Substanzen noch eine messianische Verheißung des „totalen Menschen“ (wie besonders Theologen geargwöhnt haben). Es bedeutet einfach, dass ein jeder die Möglichkeit haben soll, sich den menschlichen Reichtum anzueignen, also die Talente in sich auszubilden, die er ausbilden möchte. ${ }^{38}$ Es gibt keine Vorsortierung der Menschen nach Besitz oder Würdigkeit, Bür-

33 „Ziel der Gesellschaft ist das allgemeine Glück.“ (Verfassung der Französischen Republik vom 24. Juni 1793, Art. 1) Siehe auch: Declaration of Independence von 1776.

34 Vgl. D. McMahon, Happiness: A History, New York 2006, 312 ff.

35 G. M. McCarthy (Hg.), Marx and Aristotle. Nineteenth Century German Social Theory and Classical Antiquity, Savage 1992.

36 Eine Inspiration für Sen kam aus einer marxistischen Kritik der Wohlfahrtsökonomie, deren Doktorvater er war: H. Gintis, Alienation and Power. Towards a Radical Welfare Economics, Cambridge/ Mass. 1969.

37 Kollektive Entwicklung kennt Marx ebenfalls: „In der gewöhnlichen, materiellen Industrie [...] haben wir unter der Form [...] der Entfremdung, die vergegenständlichten Wesenskräfte des Menschen vor uns." (MEW 40, $542 \mathrm{f}$.)

38 „In diesen Arbeiten aber soll [...] nicht [...] jeder an Raffaels Statt arbeiten, sondern Jeder, in dem ein Raffael steckt, sich ungehindert ausbilden können.“ (MEW 3, 377) 
ger, Bauer, Anführer oder Künstler zu sein; jeder hat, um mit Rawls zu reden, freien Zugang zu allen „Ämtern“ beziehungsweise Lebensformen. Ob im weiteren Verlauf eine Meritokratie möglich wäre, bleibt an dieser Stelle offen; es wäre mit dem Ansatz verträglich. ${ }^{39}$ Gesagt wird jedenfalls, dass jede vieles tun können solle, wenn sie es wolle (,wie ich gerade Lust habe“). Ein liberal-egalitäres Element ist hier unabweisbar.

$\mathrm{Zu}$ Grunde liegt die aufklärerische Überzeugung der natürlichen Gleichheit der Menschen, die für Marx mit Rousseau, Kant und Adam Smith als selbstverständlich gegolten haben dürfte. Damit fällt ein elitärer Perfektionismus, wie ihn Rawls befürchtet, aus: Es ist, wenn die gesellschaftlichen Ungleichheiten der Ausbildung nicht mehr ins Gewicht fallen, kaum zu erwarten, dass die Talente in ihrer Wertigkeit derart auseinandergehen, wie es heute die Höhen der Einkommen tun. (Wohl können und sollen sie in ihrer Ausrichtung auseinandergehen, doch das heißt nicht, dass eine Ausrichtung wertvoller ist als andere.) Würde jemand für seine Entwicklung so viele gemeinschaftliche Ressourcen verbrauchen, dass andere in ihrer Entwicklung gehindert würden, verstieße dies gegen die Gleichbehandlung. Hinzu kommt, dass Marx es für wünschenswert erachtet hat, die Vergütung von der Leistung generell abzukoppeln (,Jeder nach seinen Fähigkeiten, jedem nach seinen Bedürfnissen!“; MEW 19, 22). Gleichheit bedeutet in diesem Zusammenhang also keineswegs numerische Verteilungsgleichheit, sondern ,demokratische Gleichheit“ im Sinne Andersons: die freie Ausbildung des objektiv Guten für Alle und in Gemeinschaft. ${ }^{40}$ Zwar gäbe es Unschärfen, wollte man von heute auf morgen Umverteilung an diesem Prinzip orientieren. Mit Aristoteles wäre es jedoch verfehlt, hier vorab mehr Genauigkeit zu erwarten. Warum sollte man in Zweifelsfällen darüber nicht demokratisch entscheiden? ${ }^{41}$

2. Marx' liberaler Perfektionismus. Marx hat dem Individuum, darin Humboldt ähnlich, bei der Entwicklung des „menschlichen Wesens“ einen größeren Spielraum eingeräumt als Aristoteles. ${ }^{42}$ Nun bleibt die Frage, ob Menschen, die sich ihrer eigenen Entwicklung verweigern, dazu notfalls gezwungen werden dürften. Die obige Stelle, die gegen eine nur einseitige Festlegung gerichtet ist, scheint dies auszuschließen: Wenn ich nicht dazu gezwungen werden kann, den ganzen Tag Fischer zu sein, wie soll ich dann dazu gezwungen werden, überhaupt zu fischen? Und was für das Fischen gilt, gilt für jede einzelne Tätigkeit - also für alle Tätigkeiten. Allerdings haben historische Visionen dieses Gedankens, etwa die Phalanstères im Sinne Fouriers, in denen eine solche allseitige Entwicklung schon angedacht war, dem selbstorganisierten Müßiggang wenig Platz eingeräumt. Ich bin zwar innerhalb der Phalanstère frei $\mathrm{zu}$ arbeiten, was und wo ich will, aber habe ich auch die Freiheit, gar nicht zu arbeiten?

Hier kommt nun der menschliche oder ,,wirkliche Reichtum“ ins Spiel (MEW 42, 601, 160; vgl. MEW 23, 166). Der Zwang, der bestimmte Arbeiten als ,gesellschaftlich notwen-

39 Siehe B. Priddat, Human Capital, in: Ch. Henning (Hg.), Marxglossar, Berlin 2006, 134-143.

40 „Following, Sen, I say that egalitarians should seek equality for all in the space of capabilities.“ (E. Anderson, What's the Point of Equality?, in: Ethics, 109, 1999, 316)

41 „Man [...] verlange Genauigkeit nicht bei allen Gegenständen in gleichem Maße, sondern immer nur nach Maßgabe des gegebenen Stoffes.“ (Aristoteles, Nicomachische Ethik, I.7; Hamburg 1972, 13) Where ,the concepts of equal standing and respect don't yield a determinate answer to how capabilities should be ranked, the ranking may legitimately be left up to democratic legislation“" (E. Anderson, What's the Point of Equality?, a. a. O.).

42 „Der Mensch ist im wörtlichsten Sinn ein zôon politikon, nicht nur ein geselliges Tier, sondern ein Tier, das nur in der Gesellschaft sich vereinzeln kann.“ (MEW 13, 616) Vgl. D. Thomä u. Ch. Henning, Was bleibt von der Deutschen Ideologie?, in: H. Bluhm (Hg.), Klassiker Auslegen: Die Deutsche Ideologie, Berlin 2009 (im Druck). 
dig" hinstellt und damit zu ermächtigen scheint, Individuen dazu abzuordnen, erscheint in den meisten Fällen nur innerhalb eines geldvermittelten und profitzentrierten Systems als ein solcher. „Notwendig“ sind viele Arbeiten vor allem für die Erwirtschaftung eines Profites. Was aber ist Profit? Es ist monetärer Gewinn, der von Einzelnen in Absonderung von der Gemeinschaft angeeignet wird, obwohl der Produktionsprozess insgesamt kooperativ ist. Diese Kategorie des Reichtums ist für Marx gerade das Problem - folglich kann auch der Begriff von produktiver Arbeit, der aus ihm folgt (produktiv bedeutet mehrwertproduktiv für das Kapital), nicht einfach als Ideal vorausgesetzt werden. Damit entfällt die Grundlage, bestimmte Tätigkeiten gegenüber anderen zu sanktionieren. Dies hat Auswirkungen für heutige Debatten zum Gegenleistungsprinzip (reciprocity): Ein solches Prinzip ist schwer kontrollierbar. Ein Mensch, der die meiste Zeit des Tages in seiner Kammer bastelt, kann als „unproduktiv“ für die Gesellschaft erscheinen und irgendwann doch einen großen Nutzen für alle stiften, etwa mit einer grandiosen Erfindung oder einem Roman. Die Ungenauigkeit praktischer Philosophie macht sich hier schlagend bemerkbar - ein Problem, das auch die ökonomische „Wohlfahrtstheorie" hat, die allerdings im Unterschied zu Marx überhaupt erst den Anspruch hat, Wohlfahrt zu messen. Menschlicher Reichtum hingegen ist unschätzbar; deswegen ist es der Ausbildung der Fähigkeiten dienlich, das Freiheitshindernis einer ökonomischem Berechnung menschlicher Tätigkeiten möglichst weitgehend abzubauen.

Die Frage nach der Freiheit von Arbeit verwandelt sich auf diese Weise in die Frage nach dem Status von Muße für die Entwicklung einerseits, der Begriffsbestimmung von Arbeit andererseits. Wie ein Kinderbuch beispielhaft zeigt, kann Reichtum auch dadurch gemehrt werden, dass man den ganzen Sommer über in der Sonne sitzt und träumt. Die Maus Frederick, die dies tut und von ihren Mäusekollegen darob getadelt wird, erweist sich im langen kalten Winter im Mauseloch als Dichter, der die anderen Mäuse mit seinen Sommergeschichten ,bereichert“. ${ }^{43}$ Durch diese Umbestimmung braucht man neben der freien Wahl einer Tätigkeit nicht noch eine Freiheit zur Verweigerung jeglicher Tätigkeit. ${ }^{44}$ Wirklich „nichts“ zu tun, dürfte nahezu unmöglich sein. Was man damit sagen will, ist ja, dass man nichts Produktives tut. Solch ein Urteil des „Müßiggangs“ setzt jedoch immer schon eine soziale Vorfestlegung voraus, welche Tätigkeit nützlich sei und welche nicht. Es besteht für Marx wenig Grund, diese Festlegung ausgerechnet dem Markt zu überlassen, da dieser nicht der Bedürfniserfüllung, sondern eher der Profitmaximierung dient.

Marx akzeptiert also, so können wir zusammenfassen, keinen Zwang zur Arbeit auf Grundlage des Profitmotivs. Er ist darin liberaler als der real existierende Liberalismus. ${ }^{45}$ Doch was tun im Falle „echter“ ökonomischer Knappheit, also eines manifesten Mangels nicht an profitabler Kapitalverwertung, sondern an Gebrauchswerten? Kann eine Gemeinschaft, die materielle Not leidet, von ihren Mitgliedern nicht erwarten, dass diese sich in einer bestimmten Weise einbringen, und dies notfalls auch sanktionieren? Unsere Intuition sagt uns, dass sie dies kann (vorausgesetzt, die Notlage ist echt, das heißt betrifft wirklich alle). Marx würde allerdings fordern, dass eine Gemeinschaft das auch als solche entscheiden müsste - alle, nicht nur Angehörige einer ohnehin privilegierten Oberschicht, sollen über die Verwendung der gesellschaftlichen Gesamtarbeit bestimmen. Hat der Einzelne in der Wirt-

43 Nach: L. Lionni, Frederick, du bist ja ein Dichter!, München 1990.

44 Daher war Marx keineswegs erbaut über das Buch seines Schwiegersohns Paul Lafargue, Das Recht auf Faulheit. Widerlegung des ,Rechts auf Arbeit' von 1848 [1880], Frankfurt/M. 2006.

45 Ein schlicht am Markt abgelesener Begriff von „Produktivität“ führt etwa Stuart White (The Civic Minimum, Oxford 2003, 62 f.) gegen seine egalitären Intuitionen zu einer Rechtfertigung von „workfare" (133). 
schaft ein Mitspracherecht, wäre der Zwang zumindest kein Herrschaftszwang mehr, sondern der erträglichere Zwang eines demokratischen Entscheides. Freiheitlich im Sinne realer Selbstbestimmung ist es, wenn Entscheidungen von den Menschen selbst getroffen und nicht an systemische Imperative abgetreten werden, die nur über die fragwürdige Metaphysik ökonomischer Lehrbücher einen Bezug zum Begriff der Freiheit aufweisen.

Es gibt neben der Arbeitskritik und der Forderung nach mehr ,Wirtschaftsdemokratie“ bei Marx noch eine dritte Dimension, die - erstaunlicherweise - eine liberale Perfektionismusdeutung unterstützt. Hier kommen wir auf die Musik zurück. Musik hängt metaphorologisch eng mit der menschlichen Perfektion zusammen. So läuft schon bei Leibniz eine Assoziationskette über die Ordnung, die durch die Entwicklung verwirklicht wird und in der Musik greifbar wird. ${ }^{46}$ Musik ist nicht nur eine Exzellenz des Menschen neben anderen, zudem manifestiert sie in einem ,sinnlichen Scheinen der Idee“ (Hegel) die Grundidee besonders deutlich (und so wurde sie von der Romantik als höchste Kunst angesprochen). Eine solche Verknüpfung der Musik mit der menschlichen Entwicklung findet sich auch bei Marx: Geht es um Entwicklung und „Bildung“ der menschlichen Kräfte, so spricht er von der tätigen Verwirklichung vor allem in Kunst und Musik. ${ }^{47}$ Und geht es um ,wirklich freie Arbeit“", dann spricht er vom komponieren. ${ }^{48}$ Dies zeigt erneut, dass nach diesem Reichtumsbegriff vormals als Muße gewertete Tätigkeiten als wertvoll erachtet werden. Es braucht daher keine zusätzliche Freiheit, nicht zu arbeiten.

Es ist also keine Floskel, wenn für Marx ,die freie Entwicklung eines jeden die Bedingung für die freie Entwicklung aller“ ist (MEW 4, 482). Denn nur über die „Selbstverwirklichung“ (MEW 42, 512), „die höhere Entwicklung der Individualität“, kann es zur „Entwicklung des Reichtums der menschlichen Natur" kommen (MEW 26.2, 111). Diese ist anders als in Freiheit kaum denkbar. Marx ging es, hierin Hayek ähnlich, um den Abbau von Hindernissen der Freiheit zur menschlichen Entwicklung. Darum hat er das Ausmalen künftiger Organisationsweisen geringgeschätzt: Es wäre Sache der Menschen selbst, ihre Angelegenheiten „rationell“" zu regeln (MEW 25, 828), nicht Sache philosophischer Vordenker. ${ }^{49}$ So ist es auch zu verstehen, wenn Engels intoniert: „Die Befreiung der Arbeiterklasse kann nur das Werk der Arbeiterklasse selbst sein.“ (MEW 22, 240) Perfektionismus lässt sich mit Marx nur denken, wenn man ihn praktiziert, man kann ihn nicht vorschreiben oder sich dabei vertreten lassen.

Das ist, meine ich, durchaus liberal gedacht, da die unbehinderte menschliche Tätigkeit im Vordergrund steht. Der Unterschied zum Liberalismus sans phrase ist, dass diese Freiheit nicht in Absonderung (lat. privare) von den anderen gedacht wird, sondern in der Wechselwirkung mit ihnen: „Erst in der Gemeinschaft [mit Andern hat jedes] Individuum die Mittel, seine Anlagen nach allen Seiten hin auszubilden; erst in der Gemeinschaft wird also die per-

46 G. W. Leibniz, Von der Glückseligkeit, in: Th. Leinkauf (Hg.), Leibniz, München 1996, 271 f. Musik als Bild der Ordnung zeigt Vollkommenheit an. Übrigens war ja auch Rousseau zugleich Musiktheoretiker.

47 Erst „durch den gegenständlich entfalteten Reichtum des menschlichen Wesens wird der Reichtum der subjektiven menschlichen Sinnlichkeit, wird ein musikalisches Ohr, ein Auge für die Schönheit der Form, kurz, werden erst menschlicher Genüsse fähige Sinne, Sinne, welche als menschliche Wesenskräfte sich bestätigen, teils erst ausgebildet, teils erst erzeugt“ (MEW 40, 541).

48 „Wirklich freies Arbeiten, z. B. Komponieren, ist gerade zugleich verdammtester Ernst, intensivste Anstrengung.“ (MEW 42, 512) Man bedenke die Wendung: „,man muß diese versteinerten Verhältnisse dadurch zum Tanzen zwingen, dass man ihnen ihre eigne Melodie vorsingt!“” (MEW 1, 381)

49 „Zweck der Freiheit ist, die Möglichkeit von Entwicklungen zu schaffen, die wir nicht voraussagen können.“ (F. A. Hayek, Die Ursachen der ständigen Gefährdung der Freiheit, in: Ordo, 12, Düsseldorf/München 1961, 104) 
sönliche Freiheit möglich.“ (MEW 3, 74) So führt der Gedanke der freien Entwicklung noch einmal zum Egalitarismus. Wir haben damit nicht nur eine eigenwillige Formulierung liberaler Gedanken vor uns, möglicherweise lassen sie sich so auf neue Weise alternativ begründen. Ein Liberalismus, der Entwicklung nicht schon einschließt, kann mit ihrer Förderung in Konflikt geraten. Doch ein Perfektionismus, der eine eigene Rechtfertigung liberaler Prinzipien formuliert, könnte vielleicht dazu beitragen, den alten Widerstreit der Freiheit mit der Gleichheit zu versöhnen.

3. Marx'dynamische Anthropologie. Damit ist schließlich die Frage berührt, ob der Marxsche Perfektionismus teleologisch und damit potenziell herrschaftsanfällig ist, da ein vorfestgelegtes objektives Gut sich der Selbstbestimmung der Individuen entreißen und monopolisieren ließ $\mathrm{e}^{50}$, oder ob er zieloffen ist. Wie Abschnitt II.2 zeigte, ist er eher das Letztere, da es den Individuen selbst anheim gestellt wird. Doch wie soll dieses Gut als „objektiv“ gelten, wenn doch der Spielraum der Individuen so groß ist? Das könnte immerhin subjektivistisch gelesen werden. Richtig daran ist, dass bei Marx nicht vorbestimmt ist, wohin Menschen sich entwickeln können. Doch diese Entwicklung kann gelingen oder misslingen. Spätestens in dieser Grenze meldet sich die menschliche Natur.

Menschen als „Naturwesen“ zu denken (MEW 40, 578), die hinsichtlich der Möglichkeiten ihrer Entwicklung in Freiheit leben, setzt eine komplexe Anthropologie voraus. Sie lässt sich in aller Kürze wie folgt benennen ${ }^{51}$ : Der Mensch hat für Marx wie für Hegel eine erste und eine zweite Natur. Seine „,zweite Natur“ (MEW 25, 866) ist, verkürzt gesagt, die Gesellschaft. Gesellschaftliche Verhältnisse haben eine Härte wie die Natur selbst: Sie gelten unhinterfragt und werden im Zweifelsfall durch Sanktionen gestützt. Sie kommen den Betroffenen daher natürlich vor. Darum heißt es etwa: „das menschliche Wesen ist kein dem einzelnen Individuum inwohnendes Abstraktum. In seiner Wirklichkeit ist es das ensemble der gesellschaftlichen Verhältnisse.“ (MEW 3, 6) Es gibt also etwas wie ein „Wesen des Menschen“, aber es ist gerade nicht vorgegebene Natur, sondern Ergebnis menschlicher Tätigkeiten. Es ist damit im Prinzip wandelbar. Im Bewusstsein der Kontingenz lassen sich aus dieser zweiten Natur nur schwer Wertverengungen ableiten. Es bestünde die Gefahr, bestehende Zustände (deren Problematik bekannt ist) zu totalisieren und kreative menschliche Entwicklung (deren Möglichkeit gesehen wird) zu verhindern.

Dennoch folgt daraus keine Beliebigkeit. Denn eine „,zweite Natur“ ist nur im Rahmen der ersten möglich. ${ }^{52}$ Zwar können wir diese „erste Natur“" als solche nicht erkennen. Sie kann aber gegen Zumutungen der zweiten Natur rebellieren (und das können wir erkennen, wie der Pragmatismus ähnlich formulierte). Die derart rückerschlossene „erste Natur“ des Menschen lässt viele, jedoch nicht unbegrenzt viele Möglichkeiten zu. Sie ist ein Grenzbegriff. So kann es vorkommen, dass bestimmte Organisationsweisen die Möglichkeiten der Menschen übersteigen, indem sie sie überfordern; oder sie umgekehrt einschränken, indem sie sie auf lange und stupide Arbeiten ohne Freizeit festlegen. Diese erste Natur ist also nicht positiv als Vorgabe zu formulieren, sie lässt sich nicht in dieser Weise fassen. Aber eine „negative

50 Die Gruppe um Agnes Heller nannte das einst: Dictatorship over Needs, Oxford 1983.

51 Vgl. Ch. Henning, Karl Marx, in: E. Bohlken u. Ch. Thies (Hg.), Handbuch Anthropologie. Der Mensch zwischen Natur, Kultur und Technik, Stuttgart 2009, 28-34.

52 „Auf den Menschen angewandt, [...] handelt es sich erst um die menschliche Natur im allgemeinen und dann um die in jeder Epoche historisch modifizierte Menschennatur.“ (MEW 23, 637, Fn. 63) 
Anthropologie" der ersten Natur lässt sich sehr wohl formulieren. ${ }^{53}$ Auf diese Weise lässt sich eine „Entfremdungskritik“ denken, die den Menschen nicht auf bestimmte Weisen zu sein festlegt. Ohne den Grenzbegriff der ersten Natur ließe sich diese Entfremdungskritik schwerlich formulieren, da ein unendlich formbares Wesen sich an jeden Zustand gewöhnen können müsste. Dies ist offensichtlich nicht der Fall. Kollektiv-Umerziehungsprojekte, von denen der Liberalismus keineswegs frei ist ${ }^{54}$, können an diesem „krummen Holz“ (Kant) auf grandiose Weise scheitern. Arbeitslosigkeit oder gleichgeschlechtliche Orientierungen kann man nicht mit Moralismus bekämpfen. Menschen lassen sich nicht soweit umerziehen, dass sie für Verhältnisse die Verantwortung übernehmen, für deren Zustandekommen sie nur wenig können.

Was also zeigt dieser an Marx orientierte Gegenentwurf? Dem konservativen Perfektionismus kann ein alternativer Perfektionismus entgegengesetzt werden, der die liberalen Prinzipien von Freiheit und Gleichheit nicht hintertreibt. Der Marxsche Perfektionismus ist nur ein Beispiel dafür, er ist nicht der einzig denkbare - wirkmächtige Versionen in England (bei T. H. Green, Leonard Hobhouse und Richard Tawney etwa) haben sich in bewusster Absetzung von Marx entwickelt, und auch sie sind einer näheren Betrachtung wert. Der Marxsche Perfektionismus jedenfalls ist ein nichtteleologischer „human nature“-Perfektionismus mit liberalen und egalitären Zügen. Er unterscheidet sich vom gängigen philosophischen Liberalismus und Egalitarismus durch seine Soziabilität und die Offenheit gegenüber dem antiken Glücksbegriff. Mit einer Rekonstruktion des Marxschen Perfektionismus ${ }^{55}$ lässt sich nicht nur der in Erklärungsnöte geratene liberale Egalitarismus verteidigen, er lässt sich vielleicht sogar auf neue Weise begründen.

Dr. Christoph Henning, Universität St. Gallen, Fachbereich Philosophie, Kulturwissenschaftliche Abteilung, Gatterstraße 1, 9010 St. Gallen, Schweiz

\begin{abstract}
Perfectionism is a new player in the field of political philosophy. It is often rejected by liberal philosophers for it seems to have a conservative or paternalist bias. However, this paper argues first that perfectionism can have different political tendencies: it can be both liberal and illiberal, equalizing or anti-egalitarian, and be based on both limited or open conceptions of human nature. So perfectionism is not intrinsically conservative. Secondly, the paper aims to show that a strong case can be made for a liberal and egalitarian perfectionism, based on a rereading of Karl Marx. From this perspective it is not only unnecessary to criticize perfectionism from a liberal or egalitarian perspective, perfectionism may also help in justifying liberal egalitarianism in a new way.
\end{abstract}

53 Vgl. U. Sonnemann, Negative Anthropologie. Vorstudien zur Sabotage des Schicksals, Reinbek 1969.

54 M. Bohlender, Metamorphosen des liberalen Regierungsdenkens, Weilerswist 2007.

55 Eine solche entworfen hat jüngst: D. Leopold, The Young Karl Marx: German Philosophy, Modern Politics, and Human Flourishing, Cambridge 2007; vgl. auch Ch. Henning, Was bleibt von der Marxschen Philosophie? Zu Marx' moralischem Perfektionismus, in: B. Bouvier (Hg.), Was bleibt? Karl Marx heute, Bonn 2009, 175-198. 